\title{
PREPARATION AND EVALUATION OF MICROEMULSION CONTAINING ANTIHYPERTENSIVE DRUG
}

\author{
VISHAL YADAV, PRAKASH JADHAV, KISHOR KANASE, ANJALI BODHE*, SHAILAJA DOMBE \\ Arvind Gavali College of Pharmacy, Jaitapur, Satara 415004, India \\ Email: anjali.bodheagcop@gmail.com \\ Received: 19 May 2018, Revised and Accepted: 05 Jul 2018
}

\begin{abstract}
Objective: The aim of the present study was to design novel drug delivery system containing ramipril microemulsion. Ramipril is an antihypertensive drug having very low aqueous solubility and bioavailability. Ramipril microemulsion could be used as a possible alternative to the traditional oral formulation to improve dissolution rate and hence its bioavailability with avoidance of the first-pass metabolism.

Methods: The microemulsion existence region was determined by constructing pseudoternary phase diagram and prepared by using four components orange oil, tween 80 as a surfactant, propylene glycol as co-surfactant and distilled water as the aqueous phase. The water titration method was employed for its determination. All formulated microemulsion were subjected for visual inspection, centrifugation and stability test. The all stable formulations were selected for further study.

The optimized microemulsion formulation B-9 was subjected for various evaluation parameters such as, visual inspection, stability studies, pH, viscosity measurements, electrical conductivity, content uniformity and dye solubility test.

Results: Results revealed that construction of phase diagram and use of phase titration method was a suitable technique for the preparation of microemulsion as most of the formulations were transparent. It was found that the best microemulsion result was found for ratio 2:1. The results of stress tests conclude that the optimized formulation was both physically and chemically stable for 8 mo. Also, The Fourier transform infrared (FTIR) radiation measurement and Differential scanning calorimetry (DSC) of optimized formulation indicate the compatibility of ramipril with orange oil, surfactant-tween 80 and cosurfactant-propylene glycol. From electrical conductivity $0.283 \sigma$ and staining test the prepared optimized formulation B-9 was found to be o/w type of microemulsion. The optimized B-9 formulation showed good viscosity $13.52 \pm 0.01 \mathrm{cps}$ and pH $3.21 \pm 0.02$ with highest drug content uniformity was found to be $84.98 \pm 0.02 \%$.
\end{abstract}

Conclusion: In the present study a satisfactory attempt was made to formulate a novel o/w microemulsion of ramipril which improves the gastrointestinal absorption by raising its water solubility and hence oral bioavailability is also enhanced.

Keywords: Microemulsion, Surfactants, Solubility, Phase diagram

(C) 2018 The Authors. Published by Innovare Academic Sciences Pvt Ltd. This is an open access article under the CC BY license (http://creativecommons.org/licenses/by/4.0/) DOI: http://dx.doi.org/10.22159/ijap.2018v10i5.27415

\section{INTRODUCTION}

Drug solubility enhancement is one of the most important challenges in the field of pharmaceutics. Nearly $40 \%$ of all new pharmacologically potent molecules show poor aqueous solubility, leading to their low effective concentration in biofluids and therefore poor bioavailability. Many studies have been focused on enhancing the solubility of poorly water-soluble drugs and improving bioavailability to administer them through oral route resulting in increasing their clinical efficacy. One of the most popular approach is the incorporation of the active lipophilic component into inert lipid vehicles, such as oils, surfactant dispersions, self-emulsifying formulations, emulsions, and liposomes. Microemulsion allows the incorporation of hydrophilic as well as lipophilic compound depending on their internal structure. These aggregates have been described as reservoir systems, which allow slow release of drugs, thus providing prolonged effects and avoiding high concentration in the blood [1-3].

Microemulsions (MEs) are isotropic, thermodynamically stable, transparent (or translucent) systems of oil, water, and surfactant, frequently in combination with a co-surfactant with a droplet size usually in the range of 10-100 nm. These homogeneous systems, which can be prepared over a wide range of surfactant concentration and oil to water ratio, are all fluids of low viscosity. A microemulsion as drug delivery tool show favorable properties like thermodynamic stability (long shelf-life), easy formation (zero interfacial tension and almost spontaneous formation), optical isotropy, ability to be sterilized by filtration, high surface area (high solubilization capacity) and very small droplet size. The small droplets also provide better adherence to membranes and transport drug molecules in a controlled fashion [4].

MEs have been suggested to positively influence drug absorption in a number of ways, such as protecting the incorporated drugs from oxidation and enzymatic degradation and enhancing membrane permeability and lymphatic transport. Lymphatic transport is thought to make a significant contribution to absorption from the intestine as it circumvents the hepatic first-pass effect.

The primary advantage of intestinal lymphatic drug transport is that drugs absorbed via the intestinal lymphatic system are protected from hepatic first-pass metabolism because the mesenteric lymph, in contrast to the portal blood, enters the systemic circulation directly without first passing through the liver. Therefore, intestinal lymphatic transport might have a substantial beneficial impact on drug bioavailability after oral administration, especially for drugs that are deactivated by hepatic metabolism. Mechanisms of microemulsion useful for enhancing the oral bioavailability, the comparison between oil-in-water and water-in-oil microemulsions using the single-pass intestinal perfusion [5].

We worked on drug, ramipril [(2S, 3aS, $6 \mathrm{aS})-1[(\mathrm{~S})-\mathrm{N}-[(\mathrm{S})$-carboxy-3phenyl propyl] alanylocta-hydrocyclopenta [b] pyrrole-2-carboxylic acid, 1-ethyl ester], an effective inhibitor of angiotensin-converting enzyme (ACE), prevents the conversion of AT I (Angiotensin I) to AT II (Angiotensin II). It is effective in patients suffering from hypertension, myocardial infarction, stroke, congestive heart failure and also acts as an antiproteinuric agent in children with chronic renal failure. Being a highly lipophilic compound, it has an oral bioavailability of $28-30 \%$ due to its poor aqueous solubility. This may result in dissolution related problem and low absorption and therefore the efficacy may be greatly reduced.

Due to the drawbacks stated above such as low solubility and low oralbioavailability, we tried to design an essential oil based microemulsion system as a drug delivery vehicle. A microemulsion typically consists of oil, surfactant and water in required and optimized proportions. 
These are clear, stable and isotropic mixtures. Microemulsion can be formed upon simple mixing of the components and do not require high energy conditions. The advantages of using a microemulsion are spontaneous formation, ease of manufacturing and scale-up, thermodynamic stability, improved drug solubilization of hydrophobic drugs and bioavailability [6].

In the present study, ramipril microemulsion was prepared using orange oil, tween 80 as surfactant, propylene glycol as co-surfactant and distilled water with the help of phase titration method.

\section{MATERIALS AND METHODS}

\section{Materials}

Ramipril was procured from Yarrow Chem Products, Mumbai. Orange oil and propylene glycol were obtained from Vishal Chem, Mumbai. Tween 80 was purchased from SD Lab Chemical Centre, Mumbai. All other reagents used were of analytical grade. Microemulsions are prepared by the Phase Titration Method and can be portrayed with the help of a phase diagram.

Table 1: List of instruments

\begin{tabular}{lll}
\hline S. No. & Name of the instruments & Manufacturer \\
\hline 1 & Digital pH meter & Digital Potentiometer Model EQ-601 Equip-tronics \\
2 & Laboratory centrifuge & Remi Lab-Speed 3500 \\
3 & Sonicator & Wensor-Digital Ultrasonic Cleaner WUC-1.8 L \\
4 & Brookfield viscometer & DV-E Brookfield Viscometer Model-LVDVE Mfg. Brookfield Engineering Lab \\
5 & Conductometer & Digital Conductivity meter Model-ME-976 C from Max Electronics \\
6 & Digital pan balance & Shimadzu Corporation Model-ATX224 \\
7 & UV spectrophotometer & Dynamica DB-20 Model-Halo DB-20 \\
8 & Differential scanning calorimeter & PerkinElmer 4000,PYRIS Version-11.1.0.0488, 2009, PerkinElmer, Inc. \\
9 & Infrared spectrophotometer & Shimadzu Corporation \\
10 & Orbital shaking incubator & Remi Electro-Technic Limited Model-RIS-24 BL \\
11 & Incubator & Biotechnics India \\
\hline
\end{tabular}

\section{Method}

The microemulsion of respective composition, as shown in the table 2, 3 and 4 were devised using four component orange oil as oil phase, tween 80 as a surfactant, propylene glycol as co-surfactant, and distilled water as the aqueous phase. Batches were designed for different surfactant-cosurfactant ratios (S/Cos ratio). In the present study, three $\mathrm{S} / \mathrm{Cos}$ ratios, 1:1, 2:1, 3:1, were tried.

The microemulsion containing ramipril were prepared by phase titration method constructing the phase diagram. Pseudoternary phase diagrams were constructed to investigate the effect of surfactant to cosurfactant ratio on the area of microemulsion existence region.
Pseudoternary phase diagrams of oil, water, and surfactant/cosurfactants (S/Cos) mixtures are constructed at fixed surfactant/ cosurfactant weight ratios. Phase diagrams are obtained by mixing of the ingredients, which shall be pre-weighed into glass vials and titrated with water and stirred well at room temperature. Formation of the monophasic/biphasic system is confirmed by visual inspection. In case turbidity appears followed by a phase separation, the samples shall be considered as biphasic. In case monophasic, clear and transparent mixtures are visualized after stirring; the samples shall be marked as points in the phase diagram. The area covered by these points is considered as the microemulsion region of existence [6].

Table 2: Composition of ramipril micro emulsions S/Cos ratio (1:1)

\begin{tabular}{|c|c|c|c|c|}
\hline S. No. & Ramipril (mg) & Smix & Oil & Water \\
\hline A-1 & 500 & 40 & 40 & 20 \\
\hline$A-2$ & 500 & 42 & 28 & 30 \\
\hline$A-3$ & 500 & 40 & 20 & 40 \\
\hline$A-4$ & 500 & 37 & 15 & 48 \\
\hline$A-5$ & 500 & 39 & 13 & 48 \\
\hline$A-6$ & 500 & 38 & 11 & 51 \\
\hline$A-7$ & 500 & 32 & 8 & 60 \\
\hline A-8 & 500 & 31 & 7 & 62 \\
\hline A-9 & 500 & 30 & 6 & 64 \\
\hline
\end{tabular}

Table 3: Composition of ramipril microemulsion S/Cos ratio (2:1)

\begin{tabular}{|c|c|c|c|c|}
\hline S. No. & Ramipril (mg) & Smix & Oil & Water \\
\hline B-1 & 500 & 40 & 40 & 20 \\
\hline B-2 & 500 & 45 & 30 & 25 \\
\hline B-3 & 500 & 44 & 22 & 34 \\
\hline B-4 & 500 & 40 & 16 & 44 \\
\hline B-5 & 500 & 42 & 14 & 44 \\
\hline B-6 & 500 & 38 & 11 & 51 \\
\hline B-7 & 500 & 32 & 8 & 60 \\
\hline B-8 & 500 & 31 & 7 & 62 \\
\hline B-9 & 500 & 25 & 5 & 70 \\
\hline
\end{tabular}

Phase behavior investigations of this system demonstrated the suitable approach to determine the water phase, oil phase, surfactant and co-surfactant concentration for which transparent, one phase, low viscous system form. The effect of three different surfactant co-surfactant ratios 1:1, 2:1 and 3:1 was determined in this system of orange oil/tween-80/propylene glycol and water. 
Table 4: Composition of ramipril microemulsion S/Cos ratio (3:1)

\begin{tabular}{|c|c|c|c|c|}
\hline S. No. & Ramipril (mg) & Smix & Oil & Water \\
\hline C-1 & 500 & 36 & 36 & 28 \\
\hline $\mathrm{C}-2$ & 500 & 39 & 26 & 35 \\
\hline $\mathrm{C}-3$ & 500 & 38 & 19 & 43 \\
\hline$C-4$ & 500 & 40 & 16 & 44 \\
\hline$C-5$ & 500 & 42 & 14 & 44 \\
\hline$C-6$ & 500 & 42 & 12 & 46 \\
\hline $\mathrm{C}-7$ & 500 & 36 & 9 & 55 \\
\hline $\mathrm{C}-8$ & 500 & 36 & 8 & 56 \\
\hline C-9 & 500 & 35 & 7 & 58 \\
\hline
\end{tabular}

\section{Evaluation of ramipril microemulsion}

\section{Saturation solubility of ramipril in oil, surfactant and co-surfactant}

To determine saturation solubility of ramipril shake flask method is used. An excess amount of ramipril was added to each cap vial containing $2 \mathrm{ml}$ of the vehicles. After sealing, the mixture was vortexes at maximum speed for $10 \mathrm{~min}$ in order to facilitate proper mixing of ramipril with the vehicles. Mixtures were then shaken in shaker maintained at room temperature until equilibrium (48 h). After $24 \mathrm{~h}$ the vial observed for the residue of drug, and again the excess amount of drug was added into the vial showing no residue and kept for shaking for an additional $24 \mathrm{~h}$. Then mixtures were then centrifuged at $3000 \mathrm{rpm}$ for $10 \mathrm{~min}$. The supernatants were collected into glass vials, and analysis was carried out with UVvisible spectroscopy to find the concentration of drug [8].

\section{Preparation of microemulsion}

The microemulsion existence region was determined by constructing pseudoternary phase diagram. Phase diagrams are obtained by mixing of the ingredients, which shall be pre-weighed into glass vials and titrated with distilled water and stirred well at room temperature. Formation of the monophasic/biphasic system is confirmed by visual inspection. In case turbidity appears followed by phase separation, the samples shall be considered as biphasic. In the case of the monophasic system, the clear and transparent mixtures are visualized after stirring; the samples shall be marked as microemulsion formulations [7, 15, and 17].

\section{Visual inspection}

Visual inspection was made after each addition of water to the oil and surfactant or surfactant and co-surfactant mixture. The samples were identified as microemulsion, emulsion or gel formation by visual observation $[7,8]$.

\section{Thermodynamic stability}

To overcome the problem of metastable formulation, thermodynamic stability tests were performed $[8,9]$.

\section{a) Centrifugation}

The formulation was centrifuged at $3500 \mathrm{rpm}$ for $30 \mathrm{~min}$ to ensure physical stability.

\section{b) Stress test}

These tests were done to optimize the best microemulsion formulation under extreme conditions. Stress was carried out at $4{ }^{\circ} \mathrm{C}$ and $45^{\circ} \mathrm{C}$ for $48 \mathrm{~h}$ each for a period of six cycles, followed by $25^{\circ} \mathrm{C}$ and $21^{\circ} \mathrm{C}$ for $48 \mathrm{~h}$ for about three cycles. The samples were checked for coalescence, cracking or phase separation.

\section{Measurement of $\mathrm{pH}$}

The $\mathrm{pH}$ values of the optimized formulation were measured by immersing the electrode directly into the dispersion using a calibrated pH meter (Digital Potentiometer Model EQ-601 Equip-Tronics) [7].

\section{Viscosity measurements}

The viscosity of the optimized formulation was determined as such without dilution using Brookfield Viscometer (DV-E Brookfield Viscometer Model-LVDVE).
Brookfield Viscometer consist of a cup, which is stationary and a spindle which is rotating. Different sized rotating spindles are used and immersed in test material. For liquids with low viscosity, large size spindles (large diameter and surface area) are used while for higher viscosity liquids small spindles (small diameter and surface area) are used. Rotate the spindle in the microemulsion till we get a constant dial reading on the display of the viscometer. This procedure is repeated three times for reproducible results $[7,18]$.

\section{Drug content estimation}

Microemulsion containing $100 \mathrm{mg}$ drug was dissolved in $100 \mathrm{ml}$ $0.1 \mathrm{~N} \mathrm{HCL}$ taken in volumetric flask. Then the solvent was filtered, 1 $\mathrm{ml}$ was taken in $50 \mathrm{ml}$ volumetric solution and diluted up to the mark with $0.1 \mathrm{~N} \mathrm{HCL}$ and analyzed spectrometrically at $221 \mathrm{~nm}$. The concentration of ramipril in $\mathrm{mg} / \mathrm{ml}$ was obtained by using standard calibration curve of the drug. Drug content studies were carried out in triplicate for each formulation batch $[8,18]$.

\section{Measurement of electrical conductivity}

The conductivity measurements help in determining whether the microemulsion system formed is oil-continuous or watercontinuous. The solubilization of water phase in the selected oily mixture was monitored quantitatively by measuring the electrical conductivity $(\sigma)$. The conductivity $(\sigma)$ of the formulated samples was measured using a conductivity meter (Digital Conductivity meter Model-ME-976 C from Max Electronics) [8].

\section{Staining test/dye-solubility test}

Water soluble dye, methylene blue solution of $10 \mu \mathrm{l}$ was added to the emulsion. If the continuous phase is water (o/w emulsion), the dye will dissolve uniformly throughout the system. If the continuous phase is oil (w/o emulsion), the dye will remain as cluster on the surface of the system [9].

\section{Differential scanning calorimetry (DSC)}

Thermal analysis is an important evaluation technique to find any possible interaction between the drug and excipients. Such interaction can be identified by any change in thermogram. About $1 \mathrm{mg}$ of the sample was sealed in the aluminum pan and heated at the rate of 10 ${ }^{\circ} \mathrm{C} / \mathrm{min}$, covering a temperature range of $30{ }^{\circ} \mathrm{C}$ to $300{ }^{\circ} \mathrm{C}$ under nitrogen atmosphere of flow rate $20 \mathrm{ml} / \mathrm{min}$ and DSC thermogram (PerkinElmer 4000, PYRIS Version-11.1.0.0488, 2009, PerkinElmer, Inc.) for pure drug and prepared microemulsion was obtained [16].

\section{FTIR spectroscopy}

It was determined by Fourier Transform Infrared Spectrophotometer (FTIR, Simadzu Corporation). The sample was scanned over wavelength region of 4000 to $400 \mathrm{~cm}^{-1}$ at a resolution of $4 \mathrm{~cm}^{-1}$ by dispersing sample in $\mathrm{KBr}$ and compressing into the disc by applying pressure of 5 tons for $5 \mathrm{~min}$ in a hydraulic press. The pellet was placed in light path and the spectrum was obtained [14].

\section{RESULTS AND DISCUSSION}

Determination of solubility of ramipril in different oils, surfactant and co-surfactant

For determination of solubility of ramipril, isopropyl myristate, orange oil, soybean oil, groundnut oil and coconut oil were used as 
oil phase, tween-60, tween-80 and sodium lauryl sulphate (SLS) were used as surfactants and polyethylene glycol-400 (PEG 400), polyethylene glycol-200 (PEG 200) and propylene glycol (PG) were used as co-surfactants. Each ingredient is selected based on the higher solubility of ramipril in oil, surfactant and co-surfactant. Oil and surfactant were selected on the basis of ease and spontaneous formation of an emulsion. Cosurfactant should provide very low interfacial tensions required for the formation of the microemulsion.
Required hydrophilic-lipophilic balance (HLB) for the formation of $\mathrm{o} / \mathrm{w}$ (oil in water) microemulsion was also considered while selecting oil, surfactant and co-surfactant.

The solubility of ramipril is more in orange oil, surfactant tween 80 and cosurfactant propylene glycol. The solubility of ramipril in different oils and surfactant, cosurfactant solutions are shown in table 5,6 and 7 respectively.

Table 5: Solubility study of ramipril in oils

\begin{tabular}{llll}
\hline S. No. & Phase type & Excipient & Solubility (mg/ml) \\
\hline \multirow{2}{*}{1} & \multirow{2}{*}{ Oil } & Isopropyl myristate & $0.381 \pm 0.01$ \\
& & Orange oil & $85.283 \pm 2.26$ \\
& & Soybean oil & $0.092 \pm 0.01$ \\
& & Groundnut oil & $0.104 \pm 0.01$ \\
& & Coconut oil & $0.085 \pm 0.01$ \\
\hline
\end{tabular}

(mean \pm SD; n=3)

To maintain the drug stable in its lipophilic environment oil system plays an important role. Thus different oil was chosen for our work and their solubility shown in table 5 . It was found that the solubility of ramipril was higher in orange oil $(85.283 \pm 2.26)$ as compared to other oils and so orange oil was the best vehicle for preparation of microemulsion.
Tween 80 is nonionic surfactants with high hydrophilic-lipophilic balance (values) which is a good guide for mixing water and oil into the microemulsion. As shown in table 6 tween 80 was selected as a surfactant due to its higher drug solubilizing capacity and biocompatibility than other surfactants.

Table 6: Solubility study of ramipril in surfactants

\begin{tabular}{llll}
\hline S. No. & Phase type & Excipient & Solubility $(\mathbf{m g} / \mathbf{m l})$ \\
\hline 1 & Surfactants & Tween 80 & $6.428 \pm 0.02$ \\
& Tween 60 & $0.206 \pm 0.01$ \\
& SLS & $0.445 \pm 0.03$ \\
\hline
\end{tabular}

$($ mean $\pm S D ; n=3$ )

Table 7: Solubility study of ramipril in co-surfactants

\begin{tabular}{llll}
\hline S. No. & Phase type & Excipient & Solubility (mg/ml) \\
\hline 1 & Co-surfactants & PEG 400 & $0.231 \pm 0.01$ \\
& PEG 200 & $0.142 \pm 0.01$ \\
& & Propylene glycol & $7.705 \pm 0.02$ \\
\hline
\end{tabular}

(mean $\pm S D ; n=3$ )

Co-surfactants increase the flexibility of surfactant film around the microemulsion droplet. The role of co-surfactant is to overcome the repulsive forces of similar phases and fluidity of oil and water to increase the permeability of two phases to form a microemulsion. It was observed that the solubility of ramipril was higher in propylene glycol $(7.705 \pm 0.02)$ as compared to other co-surfactants. So propylene glycol was selected as co- surfactant due to its ability to form clear and stable microemulsion formulation.

\section{Preparation of microemulsion}

Microemulsion was prepared by dissolving ramipril in orange oil. To this optimized ratio of surfactant-tween 80 and co-surfactant-propylene glycol was mixed followed by gentle mixing with distilled water.

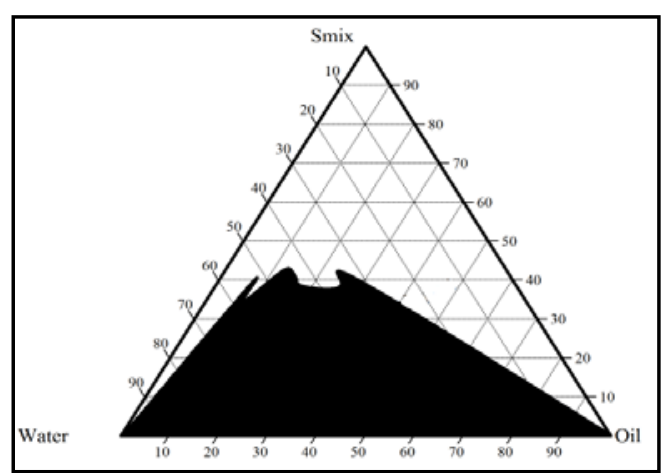

S/Cos ratio 1:1

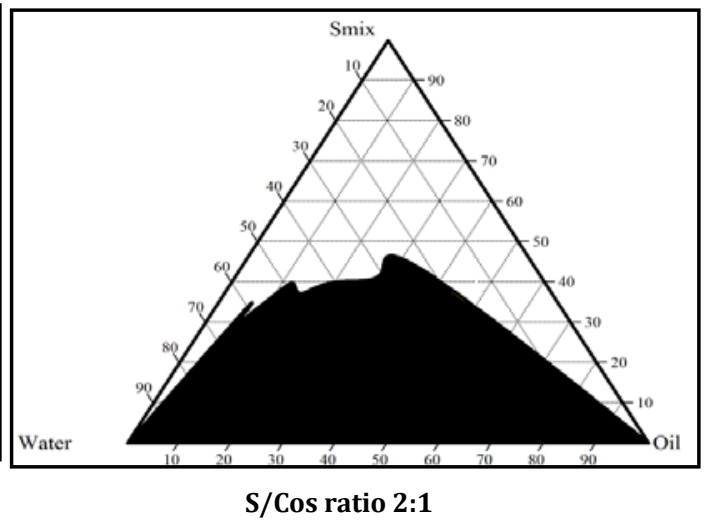




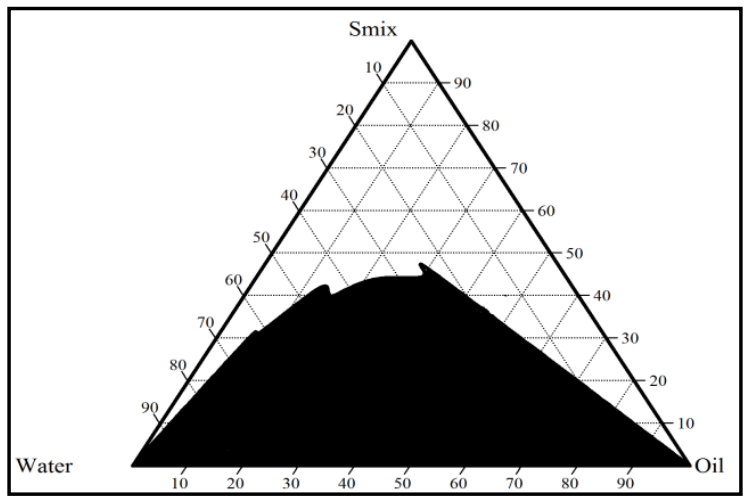

S/Cos ratio 3:1

Fig. 1: Pseudoternary phase diagrams at different $S /$ Cos ratio

A pseudoternary phase diagram of investigated quaternary system orange oil, tween 80, propylene glycol, water is presented in Fig.1. The formation of a microemulsion system (the shaded area) was observed at room temperature. From the pseudo phase diagrams, it was concluded that the highest microemulsion zone was obtained for the ratio of 3:1. It was observed that the microemulsion zone increased with increasing S/Cos ratio. There was the very slight difference in microemulsion zone obtained for $\mathrm{S} /$ Cos ratio $3: 1$ and $2: 1$. The phase study revealed that the maximum proportion of water is incorporated in the microemulsion system when the S/Cos ratio was 2:1. Increased content of water and decreased the content of surfactant-co-surfactant and oil provide a greater opportunity for the solubilization of ramipril which is an added advantage for less viscous and transparent micro emulsion.

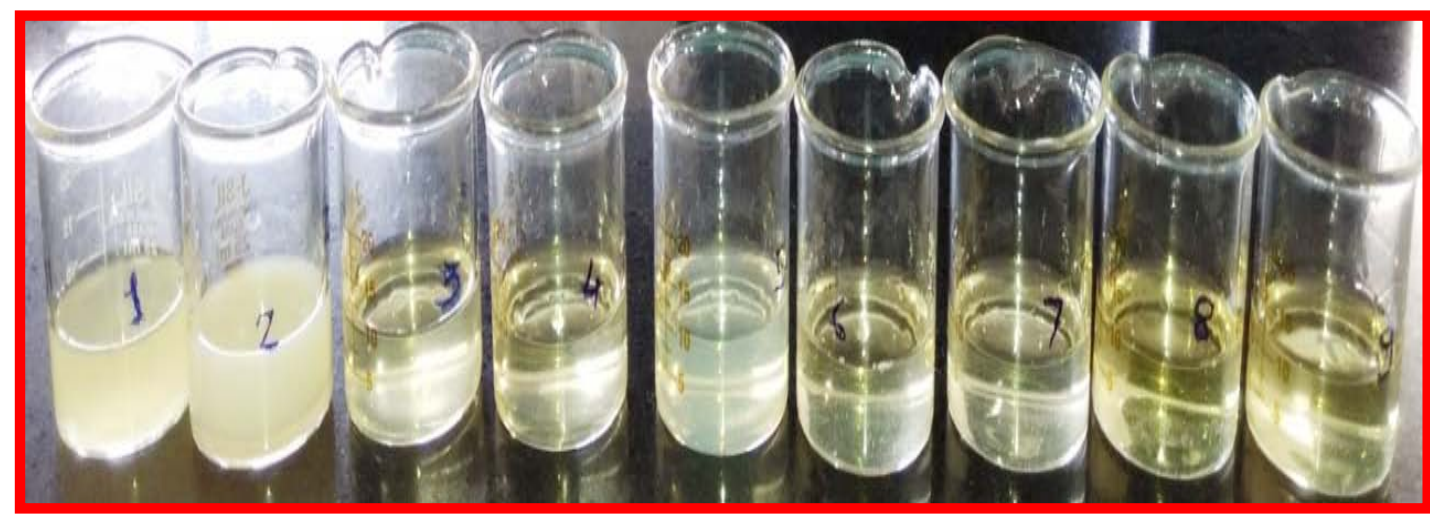

Fig. 2: Microemulsion preparation $S / C o s$ ratio 2:1

The developed microemulsion containing ramipril, orange oil, tween 80 , propylene glycol and water was found to be transparent at increased water content and decreased surfactant and oil content. At first, the microemulsion showed milky appearance but at increased water content and optimum $\mathrm{S} / \mathrm{Cos}$ ratio $2: 1$ it showed transparent flowable microemulsion.

\section{Visual inspection}

The samples were identified as microemulsions when they appear as transparent/translucent and easily flowable liquid. The samples were identified as emulsions when they appeared as milky or turbid liquids.

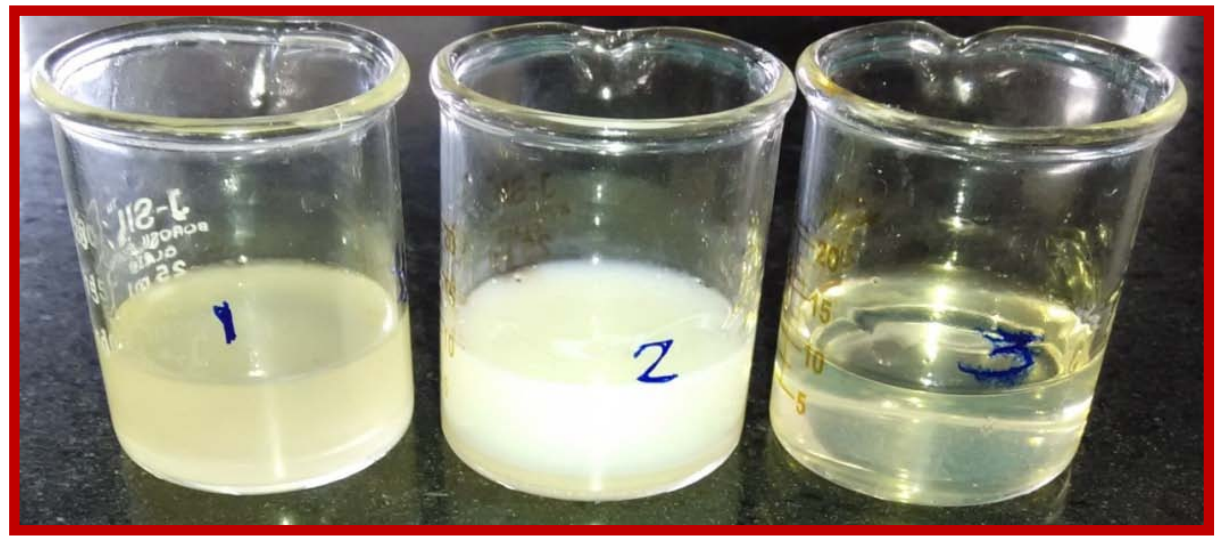

Fig. 3: Difference between emulsion and microemulsion formulations 
In fig. 3 first two formulations were found to be milky and turbid so considered as emulsions with two phases. And the last one that is the third formulation was transparent and easily flowable liquid so considered as microemulsion with only one phase.

\section{Thermodynamic stability}

Thermodynamic stability tests were performed by

\section{a) Centrifugation}

Formulations A-1 to A-3, B-4 to B-6 and C-1 to C-3 clearly separated into two phases after centrifugation, which was milky, white in appearance considered as emulsions and rejected. The centrifugation tests revealed that microemulsion formulations A-4 to $\mathrm{A}-9, \mathrm{~B}-1$ to $\mathrm{B}-3, \mathrm{~B}-7$ to $\mathrm{B}-9$ and $\mathrm{C}-4$ to $\mathrm{C}-9$ were remained homogenous without any phase separation throughout the test indicates good physical stability and taken for further study.

\section{b) Stress test}

As per following data, only eleven formulations passed through different stress conditions as shown in table 8. Formulation A-4, A-5, A-8 and A-9 were passed the centrifugation and stress test. Microemulsion formulations B-3, B-8 and B-9 showed good results with better stability. Similarly, microemulsion formulations C-5, C-6, C-8 and C-9 were stable in all temperature conditions. From all these stable formulations the microemulsions containing a high content of surfactant-co-surfactant were rejected.

Table 8: Observations of stress tests

\begin{tabular}{|c|c|c|c|c|}
\hline \multirow{2}{*}{ Formulations } & \multicolumn{4}{|c|}{ Observations of stress tests } \\
\hline & Centrifugation & 25 to $30^{\circ} \mathrm{C}$ & $45^{\circ} \mathrm{C}$ & $4^{\circ} \mathrm{C}$ \\
\hline A-4 & $\checkmark$ & $\checkmark$ & $\checkmark$ & $\checkmark$ \\
\hline$A-5$ & $\checkmark$ & $\checkmark$ & $\checkmark$ & $\checkmark$ \\
\hline A- 6 & $\checkmark$ & $\checkmark$ & $\checkmark$ & $\mathrm{x}$ \\
\hline A-7 & $\checkmark$ & $\checkmark$ & $\mathrm{x}$ & $\mathrm{x}$ \\
\hline A-8 & $\checkmark$ & $\checkmark$ & $\checkmark$ & $\checkmark$ \\
\hline A-9 & $\checkmark$ & $\checkmark$ & $\checkmark$ & $\checkmark$ \\
\hline B-1 & $\checkmark$ & $\mathrm{x}$ & $\mathrm{x}$ & $\checkmark$ \\
\hline B-2 & $\checkmark$ & $\mathrm{x}$ & $\mathrm{x}$ & $\checkmark$ \\
\hline B-3 & $\checkmark$ & $\checkmark$ & $\checkmark$ & $\checkmark$ \\
\hline B-7 & $\checkmark$ & $\checkmark$ & $\checkmark$ & $\mathrm{x}$ \\
\hline B-8 & $\checkmark$ & $\checkmark$ & $\checkmark$ & $\checkmark$ \\
\hline B-9 & $\checkmark$ & $\checkmark$ & $\checkmark$ & $\checkmark$ \\
\hline $\mathrm{C}-4$ & $\checkmark$ & $\checkmark$ & $\mathrm{x}$ & $\checkmark$ \\
\hline C-5 & $\checkmark$ & $\checkmark$ & $\checkmark$ & $\checkmark$ \\
\hline C- 6 & $\checkmark$ & $\checkmark$ & $\checkmark$ & $\checkmark$ \\
\hline C-7 & $\checkmark$ & $\mathrm{x}$ & $\mathrm{x}$ & $\checkmark$ \\
\hline C-8 & $\checkmark$ & $\checkmark$ & $\checkmark$ & $\checkmark$ \\
\hline C-9 & $\checkmark$ & $\checkmark$ & $\checkmark$ & $\checkmark$ \\
\hline
\end{tabular}

Thus A-9, B-9 and C-9 formulation serves as the best microemulsion formulations with no phase separation, creaming or flocculation and was taken for further characterization. The results of the stress tests concluded that the formulations were both physically and chemically stable. The formulations which showed the very high content of surfactant were rejected.

Table 9: Viscosity, pH and content uniformity

\begin{tabular}{lllll}
\hline S. No. & Formulation & Viscosity(cps) & pH & Content uniformity \\
\hline 1 & A -9 & $16.62 \pm 0.02$ & $3.48 \pm 0.01$ & $72.48 \pm 0.02$ \\
2 & B-9 & $13.52 \pm 0.01$ & $3.21 \pm 0.02$ & $84.98 \pm 0.02$ \\
3 & C-9 & $18.23 \pm 0.01$ & $3.63 \pm 0.01$ & $69.52 \pm 0.01$ \\
\hline
\end{tabular}

$($ mean \pm SD; $n=3$ )

\section{Evaluation parameters of formulations}

The physicochemical characteristics of developed microemulsion appear in table 9. From the above result, it was found that formulations showed increased $\mathrm{pH}$ with increased $\mathrm{S} / \mathrm{Cos}$ ratio. It was observed that formulation B-9 showed large content uniformity (84.98\%) having viscosity $13.52 \mathrm{cps}$ and $\mathrm{pH} 3.21$ than other two formulations A-9 and C-9. B-9 formulation showed more water quantity with less surfactant having large microemulsion region. So formulation B-9 is considered as an optimized microemulsion.

\section{Measurement of electrical conductivity}

The conductivity of the optimized microemulsion (B-9) as determined by the conductivity meter was found to be $0.283 \sigma$. From electro conductivity study it can be concluded that the system is of o/w type.

\section{Staining test/dye-solubility test}

Water soluble dye, methylene blue solution was added to optimized microemulsion (B-9), the dye will dissolve uniformly throughout the system, so the continuous phase was water. Hence the optimized formulation B-9 was found to be o/w type of microemulsion is shown in fig. 4.

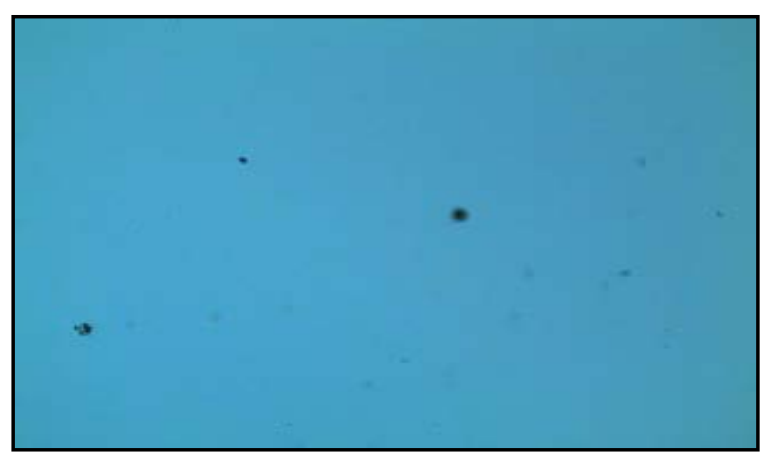

Fig. 4: Staining of optimized microemulsion (B-9) 


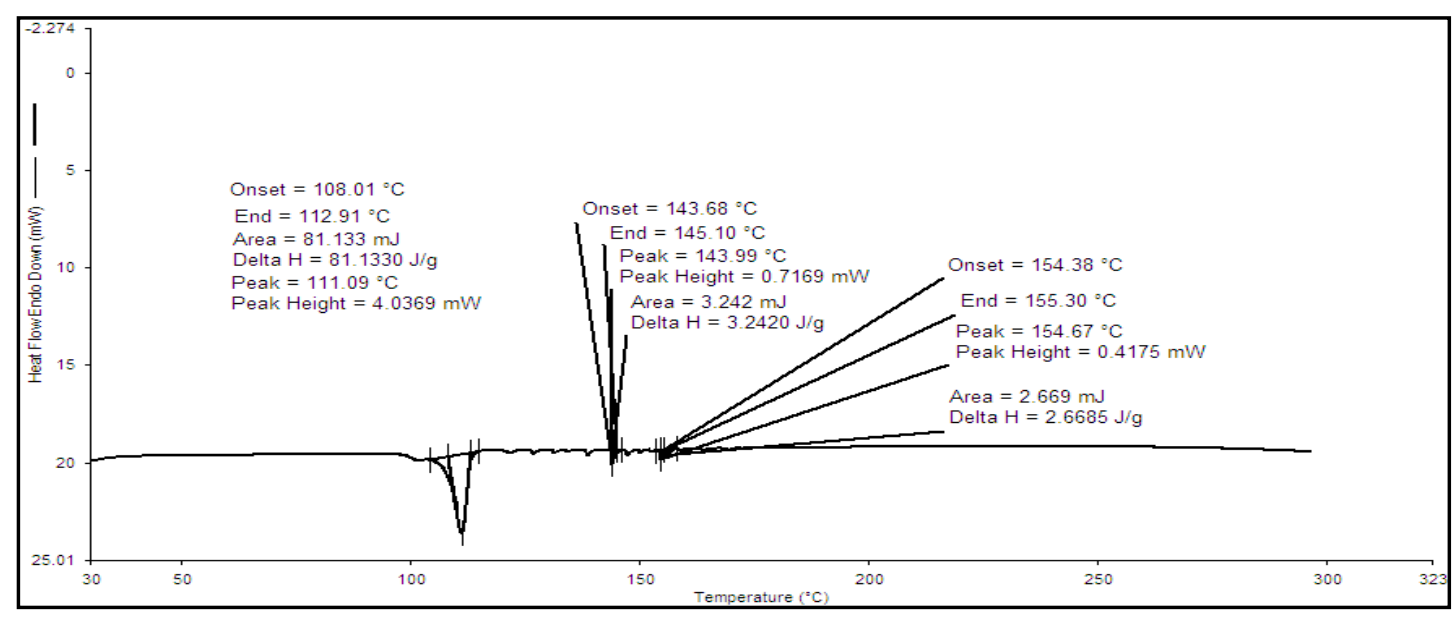

Fig. 5: DSC of pure ramipril

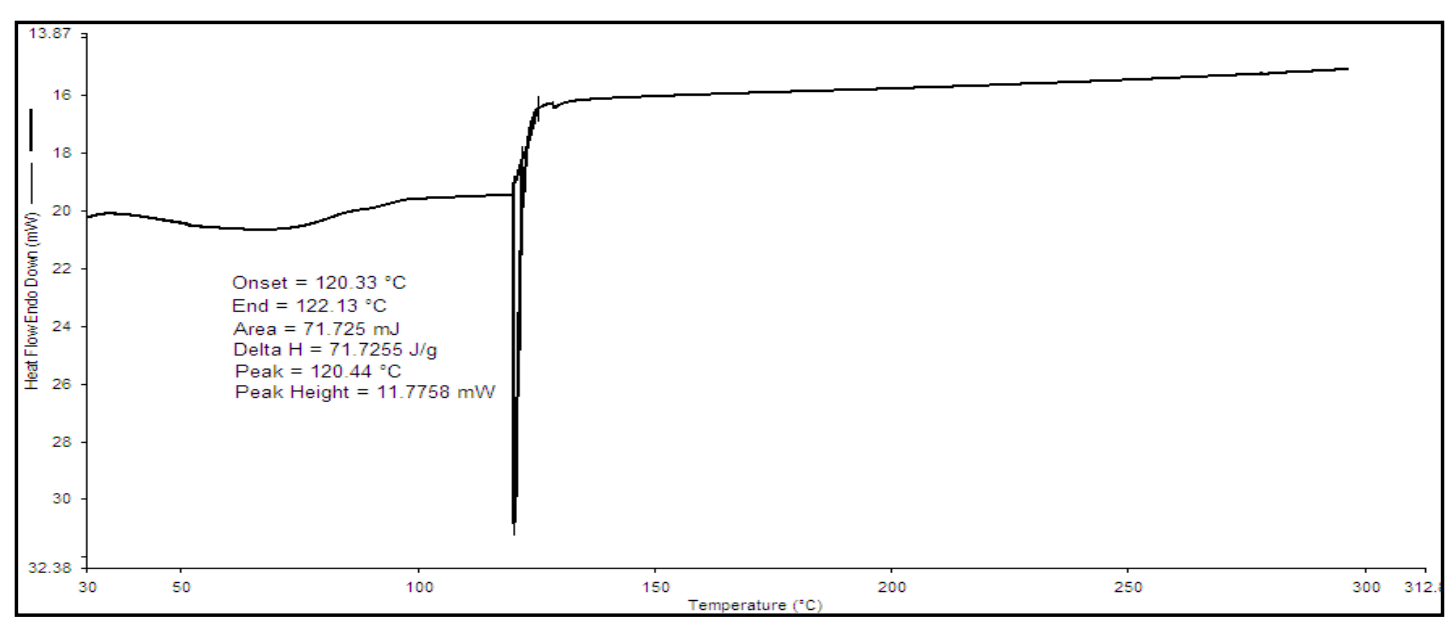

Fig. 6: DSC of optimized formulation B-9

Thermograph of ramipril is shown in fig. 5. Melting transition of ramipril was observed from $108.01{ }^{\circ} \mathrm{C}$ (Onset) to $112.98{ }^{\circ} \mathrm{C}$ (Endset). Sharp melting transition of ramipril was observed at $111.09{ }^{\circ} \mathrm{C}$. In the optimized formulation fig. 6, drug and excipients melting endotherm was observed from $120.33{ }^{\circ} \mathrm{C}$ (Onset) to $122.13{ }^{\circ} \mathrm{C}$ (Endset). Sharp melting transition of Ramipril was observed at $120.44{ }^{\circ} \mathrm{C}$. In case of optimized formulation, B-9 drug peak is shifted to slightly lower temperature and decreases the intensity of peak which may be due to baseline shifting. There was no much difference in the melting point of the drug in optimized B-9 microemulsion thermographs, shown in fig. 7.

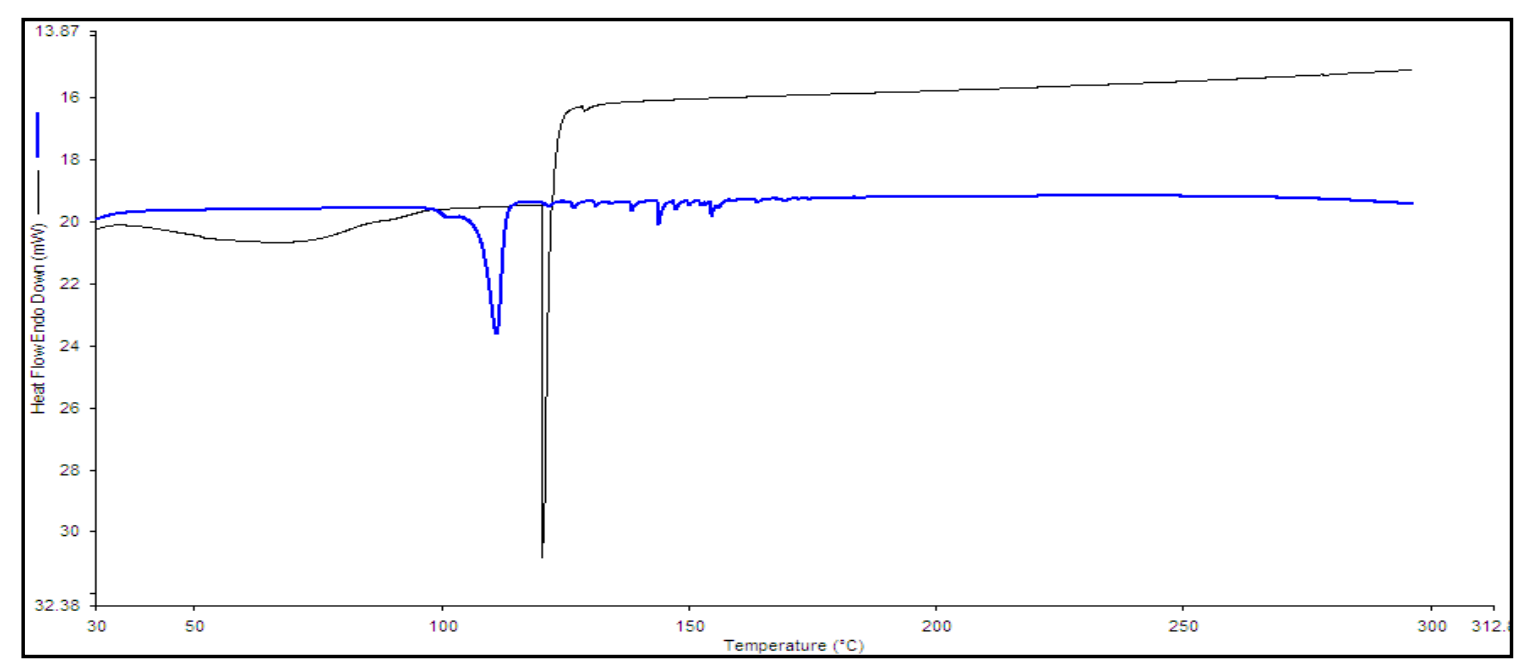

Fig. 7: Comparison of pure drug and optimized formulation B-9 


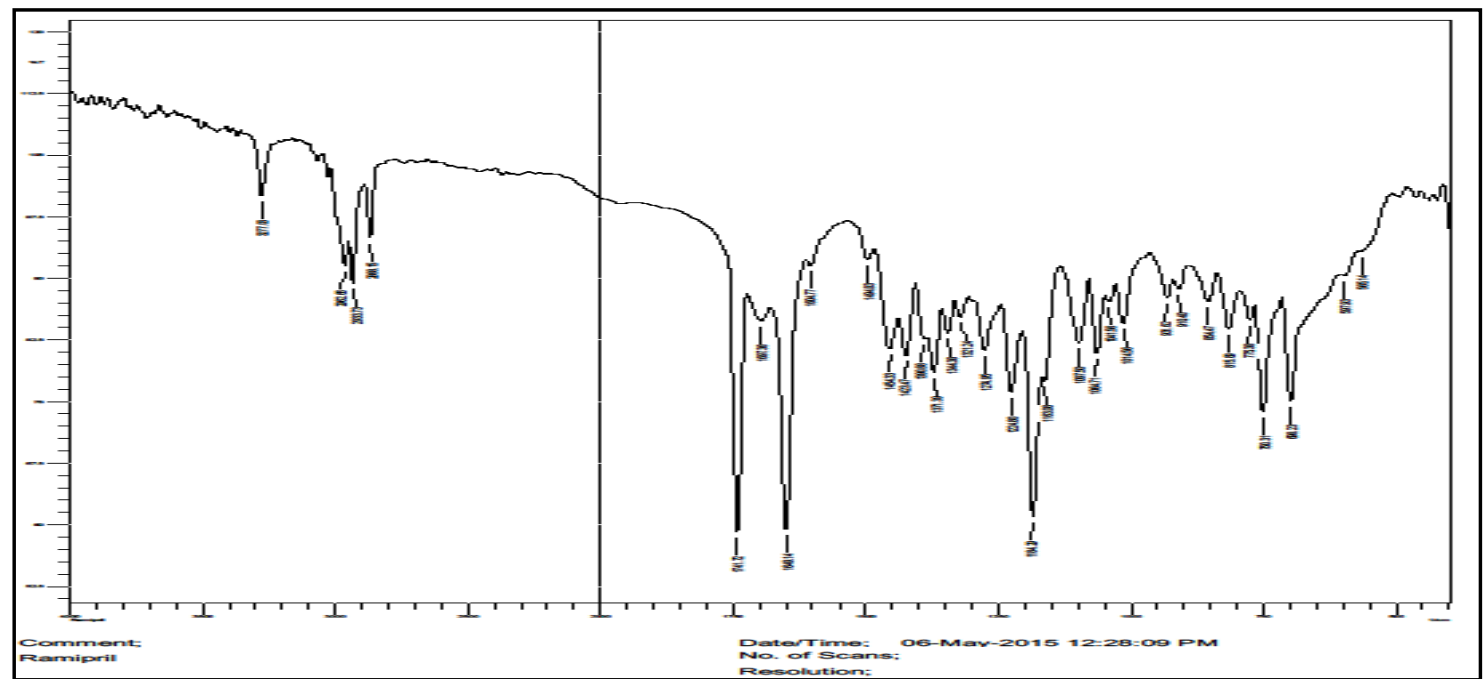

Fig. 8: FTIR of ramipril

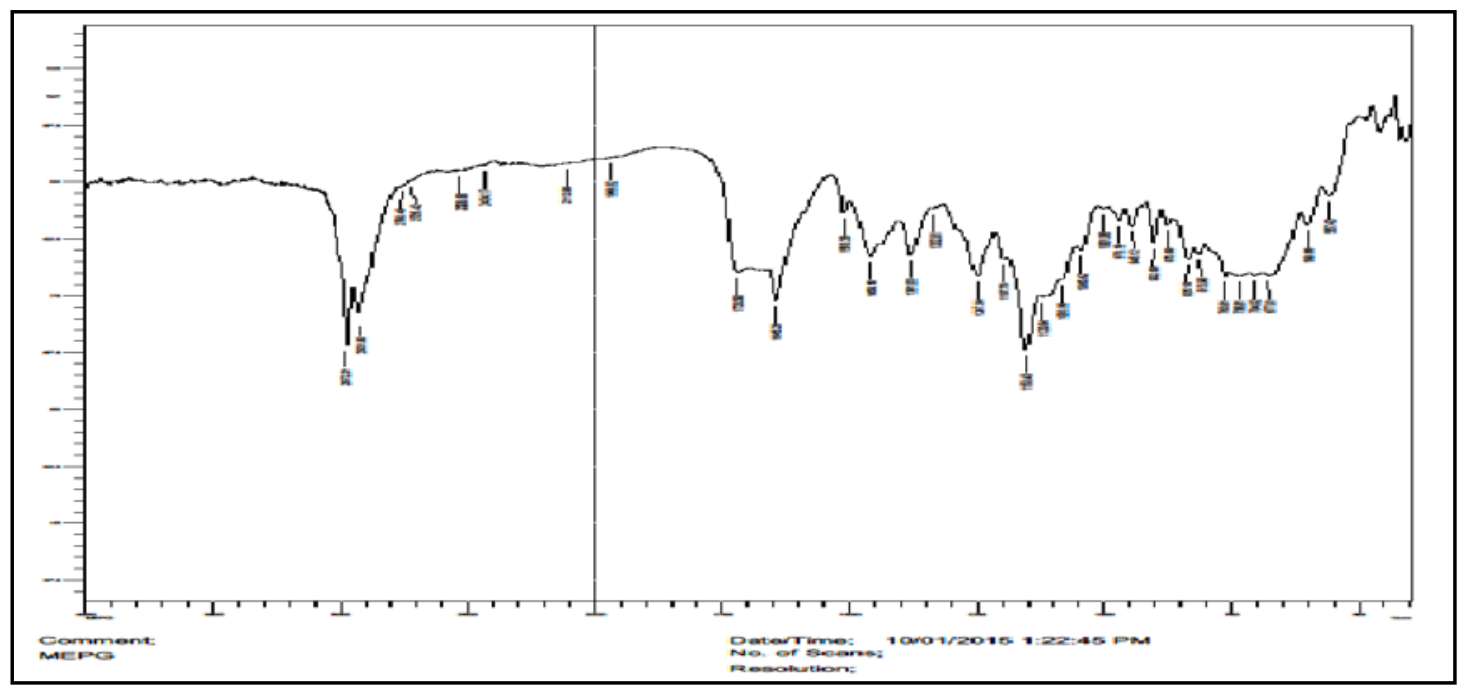

Fig. 9: FTIR of optimized microemulsion B-9

FTIR studies revealed that there was no appearance of any new peak and disappearance of existing peaks, which indicated that there was no chemical interaction between the drug and excipients used. The characteristic- $\mathrm{NH}$ and- $\mathrm{OH}$ stretching at $3278.99 \mathrm{~cm}^{-1},-\mathrm{CH}$ aromatic stretching at $2929.87 \mathrm{~cm}^{-1},-\mathrm{CH}$ aliphatic stretching at $1741.72 \mathrm{~cm}^{-1}$,$\mathrm{C}=0$ of acid at $-\mathrm{C}=0$ of acid at $1651.07 \mathrm{~cm}^{-1}$ ester group at 1498.69 and $1450.47 \mathrm{~cm}^{-1}$ were identified in all the spectra. All the characteristic peaks of Ramipril were observed in the IR spectra of physical drug and optimized formulation B-9 during the investigation of compatibility study which is shown in fig. 9. Hence IR spectroscopy results showed that the drug was compatible with selected excipients and was stable in optimized B-9 microemulsion.

\section{CONCLUSION}

The developed microemulsion containing orange oil was found to be a suitable vehicle to formulate ramipril microemulsion. Microemulsion containing orange oil, tween 80 , propylene glycol and water provide a useful dosage form for poorly water-soluble drug ramipril. By the construction of the phase diagram, it was found that best microemulsion results were found for ratio $2: 1$. In the present study optimized formulation, B-9 was transparent and easily flowable liquid. The results of stress tests concluded that the optimized formulation was physically and chemically stable for 8 mo. From electrical conductivity $(0.283 \mu \mathrm{S} / \mathrm{cm})$ and staining test, it was found that the optimized formulation B-9 was o/w type of microemulsion having content uniformity $84.98 \%$, viscosity $13.52 \mathrm{cps}$ and $\mathrm{pH} 3.21$. Also, FTIR and DSC study of optimized formulation indicate the compatibility of ramipril with orange oil, surfactant-tween 80 and cosurfactantpropylene glycol. Thus the study confirmed that microemulsion formulation can be used as a possible alternative to the conventional oral formulation of ramipril to improve its solubility and oral absorption. It was concluded that the high content of water gives us $\mathrm{o} / \mathrm{w}$ microemulsion with low viscosity having more absorption inside the body and lower content of surfactant reduces the side effects.

\section{AUTHORS CONTRIBUTIONS}

All the author have contributed equally

\section{CONFLICT OF INTERESTS}

Declared none

\section{REFERENCES}

1. C Srinivas, S Vanitha Sagar. Enhancing the bioavailability of simvastatin using microemulsion drug delivery system. Asian J Pharm Clin Res 2012;5:134-9. 
2. Faizi Muzaffar, UK Singh, Lalit Chauhan. Review on microemulsion as futuristic drug delivery. Int J Pharm Pharm Sci 2013;5:39-53.

3. Ramaiyan Dhanapal. A review-microemulsion. Asian J Pharm Res 2012;2:23-9.

4. Sarkhejiya Naimish A, Nakum Mayur A, Patel Vipul P, Atara Samir A, Desai Thusarbindu R. Emerging trend of the microemulsion in formulation and research. Int Bull Drug Res 2010;1:54-83.

5. Tian Tian Tang, Xiong Bin Hu, De Hua Liao, Xin Yi Liu, Da Xiong Xiang. Mechanisms of microemulsion enhancing the oral bioavailability of puerarin: comparison between oil-in-water and water-in-oil microemulsions using the single-pass intestinal perfusion method and a chylomicron flow blocking approach. Int J Nanomed 2013;8:4415-26.

6. Sushama Talegaonkar, Adnan Azeem, Farhan J Ahmad, Roop K Khar, Shadab A Pathan, Zeenat I Khan. Microemulsions: a novel approach to enhanced drug delivery. J Korean Neurosurg Soc 2008;2:238-57.

7. M Joyce Nirmala, Srinivas Allankib, Amitava Mukherjeea, N Chandrasekarana. Enhancing the solubility of ramipril using a new essential oil based microemulsion system. Int J Pharm Pharm Sci 2013;5:322-3.

8. M Joyce Nirmala, N Chandrasekaran, Amitava Mukherjee. Enhanced solubilization of aqueous insoluble anti-hypertensive drug. Int J Pharm Pharm Sci 2012;4:366-8.

9. Divya A, Ch Praveen Kumar, K Gnanaprakash, M Gobinath Design, formulation and characterization of tenofovir microemulsion as oral drug delivery. Int J Pharm Rev Res 2014;4:1-5.
10. Surjyanarayan Mandal, Snigdha S Mandal. Microemulsion drug delivery system: a platform for improving the dissolution rate of the poorly water-soluble drug. Int J Pharm Sci Nanotechnol 2011;3:1214-9.

11. Neha Tyagi, NV Sateesh Madhav. Formulation and evaluation of zidovudine microemulsion using a novel biopolymer from the seeds of buchanania llanzan. Int J Biopharm 2012;3:40-3.

12. Mandal Surjyanarayan, Mandal Snigdha S, Surti Naazneen, Patel Vandana B Parul, Arogya Seva Mandal. Design and development of squinavir microemulsion for the oral bioavailability enhancement. Int $J$ Pharm Tech Res 2009;1:1442-8.

13. Jha SK, Dey S, Karki R. Department of Pharmaceutics, a microemulsions-potential carrier for improved drug delivery. Asian J Biomed Pharm Sci 2011;1:5-9.

14. Indian Pharmacopoeia, Government of India Ministry of Health and Family Welfare. Vol. III; 2014. p. 2639.

15. Shah RR, Magdum CS, Wadkar KA, Naikwade NS. Fluconazole topical microemulsion: preparation and evaluation. Res J Pharm Tech 2009;2:353-8.

16. Eskandar Moghimipour, Anayatollah Salimi, Soroosh Eftekhari. Design and characterization of microemulsion systems for naproxen. Adv Pharm Bull 2013;3:63-71.

17. Anindya Hana Iradhati, Mahdi Jufri. Formulation and physical stability test of griseofulvin microemulsion gel. Int J App Pharm 2017;9:23-6.

18. Vishal Yadav, Prakash Jadhav, Shailaja Dombe, Anjali Bodhe, Pranali Salunkhe. Formulation and evaluation of microsponge gel for topical delivery of the antifungal drug. Int J Appl Pharm 2017;9:30-7. 